International Mathematical Forum, Vol. 9, 2014, no. 8, 367 - 375

HIKARI Ltd, www.m-hikari.com

http://dx.doi.org/10.12988/imf.2014.312227

\title{
On Fuzzy Congruences of a Type-B Semigroup $\mathbf{I}^{1}$
}

\author{
Chunhua Li \\ School of Basic Science, East China Jiaotong University \\ Nanchang, Jiangxi 330013, P.R. China
}

Copyright (c) 2014 Chunhua Li. This is an open access article distributed under the Creative Commons Attribution License, which permits unrestricted use, distribution, and reproduction in any medium, provided the original work is properly cited.

\begin{abstract}
The main aim of this paper is to study fuzzy congruences of typeB semigroups. After obtaining some properties and characterizations of fuzzy good congruences on adequate semigroups, we consider fuzzy good congruences on type-B semigroups. Finally, we prove a theorem giving equivalent conditions on fuzzy cancellative congruences of such semigroups.
\end{abstract}

Mathematics Subject Classification: 06F05; 20M10

Keywords: Fuzzy relations; Fuzzy good (cancellative) congruence; Idempotentseparating; Adequate semigroups; Type-B semigroups

\section{Introduction}

The relations $\mathcal{L}^{*}$ and $\mathcal{R}^{*}$ are defined on a semigroup $S$ by the rule that the elements $a$ and $b$ of $S$ are related by $\mathcal{L}^{*}$ [ resp. $\mathcal{R}^{*}$ ] on $S$ if and only if they are related by $\mathcal{L}$ [ resp. $\mathcal{R}$ ] in some oversemigroup of $S$ ( see, [3] ). The intersection of the equivalence relations $\mathcal{L}^{*}$ and $\mathcal{R}^{*}$ is denoted by $\mathcal{H}^{*}$. According to Fountain [5], a semigroup $S$ is called rpp [ resp. lpp ] if and only if each $\mathcal{L}^{*}$ class [ resp. $\mathcal{R}^{*}$ class ] contains at least one idempotent. A semigroup $S$ is called abundant if it is both rpp and lpp. An rpp [ resp. a lpp ] semigroup in which the idempotents commute is right adequate [ resp. left

\footnotetext{
${ }^{1}$ This work is supported by the National Science Foundation of China(No. 11261018; 11061014), the Natural Science Foundation of Jiangxi Province ( No. 20122BAB201018) the JiangXi Educational Department Natural Science Foundation of China (No. KJLD12067).
} 
adequate ]. A semigroup $S$ is called adequate if and only if it is both right and left adequate. For convenience, we denote by $a^{+}\left[a^{*}\right]$ a typical idempotent $\mathcal{R}^{*}$-related [ $\mathcal{L}^{*}$-related ] to $a$. And $E(S)$ denotes the set of idempotents of $S$. It can be easily seen that each $\mathcal{L}^{*}$ class [ resp. $\mathcal{R}^{*}$ class ] of an adequate semigroup $S$ contains exactly one idempotent, and that $(a b)^{+}=\left(a b^{+}\right)^{+}$and $(a b)^{*}=\left(a^{*} b\right)^{*}$ for all $a \in S$. A right adequate semigroup $S$ is called right type- $B$, if it satisfies:

(B1) for all $e, f \in E\left(S^{1}\right), a \in S,(e f a)^{*}=(e a)^{*}(f a)^{*}$;

(B2) if for all $a \in S, e \in E(S), e \leq a^{*}$, then there is an element $f \in E\left(S^{1}\right)$ such that $e=(f a)^{*}$, where " $\leq$ " is a natural partial order on $E(S)$ (i.e., $(\forall e, f \in E(S)) \quad e \leq f \Leftrightarrow e=e f=f e \quad($ see,$[9]))$.

Dually, we can define a left type- $B$ semigroup. A semigroup $S$ is called type- $B$ if and only if it is both right and left type-B ( see, [4] ).

Let $X$ be a non-empty set. A fuzzy subset $f$ of $X$ is a function of $X$ into the closed interval [0,1]. In 1971, Rosenfeld [18] explained the notion of a fuzzy subgroup of a group with the notion of fuzzy subsets of a set. The development of fuzzy abstract algebra has since been greatly inspired by the results of the research $[2,8,14,15,19,20,21,22]$. Following the formulation of fuzzy groups by Rosenfeld, many researchers have been engaged in extending the concepts and results of pure algebra to the broader framework of the fuzzy setting, although not all results in algebra can be fuzzified. As we know, the concepts of fuzzy equivalent relations on a set were introduced by Murali [14] and Nemitz [15], respectively. Samhan [19] defined fuzzy congruence relations on semigroups. In 1997, Nobuaki kuroki [8] studied fuzzy congruences on inverse semigroups (see,[17]). As a generalization of inverse semigroups in the range of abundant semigroups, Fountain [4] introduced adequate semigroups. After that, various classes of adequate semigroups are researched (see, $[1,6,10$, $11,12]$ ). Motivated by the study of fuzzy congruences in groups, semigroups, and ordered semigroups, and also motivated by N. Kuroki and Y. Tan's works in a regular semigroup (i.e., for each element $a$ of a semigroup $S$, there exists an element $x$ of $S$ such that $a=\operatorname{axa}$ (see, [7]) ) in terms of fuzzy subsets, we attempt in this paper to study the fuzzy congruences on a type-B semigroup $S$ in detail. As we know, an arbitrary congruence $\rho$ on a regular semigroup $S$ is regular $\mathcal{L}$-class preserving and regular $\mathcal{R}$-class preserving. That is, for $a, b \in S, a \mathcal{L} b[\operatorname{resp} . a \mathcal{R} b]$ implies that $a \rho \mathcal{L} b \rho[\operatorname{resp} . a \rho \mathcal{R} b \rho]$. But it is not true for a congruence $\rho$ on an abundant semigroup $S$ that $a \mathcal{L}^{*} b$ [ resp. $a \mathcal{R}^{*} b$ ] implies that $a \rho \mathcal{L}^{*} b \rho\left[\right.$ resp. $\left.a \rho \mathcal{R}^{*} b \rho\right]$. In fact, only a good congruence $\rho$ on an abundant semigroup $S$ is abundant $\mathcal{L}^{*}$-class preserving and abundant $\mathcal{R}^{*}$-class preserving. Li and Liu [12] introduced the notion of a fuzzy good congruence relation on abundant semigroups which preserve the Green's *-relation $\mathcal{L}^{*}$ and $\mathcal{R}^{*}$ with respect to the binary operation $*$ ( see, [13] ). It is a key notion to depicting the properties and characterizations of abundant semigroups( the 
reader may consult [12]).

In this paper, we shall consider fuzzy good congruences on type-B semigroups by using the results of $\mathrm{Li}$ and $\mathrm{Liu}$ in [12]. Our main result is to show that $S / \mu$ is itself type-B with respect to the binary operation $*$ for any fuzzy good congruence $\mu$ on a type-B semigroup $S$.

\section{Preliminaries}

We follow the notions adopted in $[3,4,5,13,16]$. First, we state some known results and notations which will be frequently used throughout the paper.

Lemma 1.1. [3] Let $S$ be a semigroup and $a, b \in S$. Then the following statements are equivalent:

(1) $a \mathcal{L}^{*} b\left(a \mathcal{R}^{*} b\right)$;

(2) for all $x, y \in S^{1}$, ax $=a y[x a=y a]$ if and only if $b x=b y[x b=y b]$.

Corollary 1.2. [3] Let $S$ be a semigroup and $e^{2}=e, a \in S$. Then the following statements are equivalent:

(1) $a \mathcal{L}^{*} e\left(a \mathcal{R}^{*} e\right)$;

(2) $a e=a[e a=a]$ and for all $x, y \in S^{1}$, ax $=a y[x a=y a]$ implies ex $=e y$ $[x e=y e]$.

Evidently, $\mathcal{L}^{*}$ is a right congruence while $\mathcal{R}^{*}$ is a left congruence. In an arbitrary semigroup, we have $\mathcal{L} \subseteq \mathcal{L}^{*}$ and $\mathcal{R} \subseteq \mathcal{R}^{*}$. But for regular elements $a, b$, we get $a \mathcal{L}^{*} b\left[a \mathcal{R}^{*} b\right]$ if and only if $a \mathcal{L} b[a \mathcal{R} b]$. As in [9], Lawson define a natural partial order relation " $\leq$ " on an abundant semigroup $S$ as follows: $(\forall a, b \in S) a \leq b$ if and only if there are idempotents $e, f \in S$, such that $a=e b=b f$.

We recall from [3] that a semigroup homomorphism $\phi: S \rightarrow T$ is a good homomorphism, if for all elements $a, b$ of $S, a \mathcal{L}^{*}(S) b$ implies $a \phi \mathcal{L}^{*}(T) b \phi$, and $a \mathcal{R}^{*}(S) b$ implies $a \phi \mathcal{R}^{*}(T) b \phi$. We say that a congruence $\rho$ on a semigroup $S$ is a good congruence if the natural homomorphism from $S$ onto $S / \rho$ is good. As defined in [16], a congruence $\rho$ on a semigroup $S$ is an idempotent-separating congruence, if for all $e, f \in E(S)$ the equality $e \rho=f \rho$ implies that $e=f$. A congruence $\rho$ on a semigroup $S$ is a cancellative congruence if $S / \rho$ is a cancellative semigroup. Evidently, a cancellative congruence is good. From [3], we quote

Lemma 1.3. [3] Let $S$ be an abundant semigroup. Then

(1) Let $\rho$ be a congruence on $S$ and $a \in S$. Then $\rho$ is good if and only if $a \rho \mathcal{L}^{*}(S / \rho) a^{*} \rho$ and $a \rho \mathcal{R}^{*}(S / \rho) a^{+} \rho$; 
(2) $\eta_{l}=\left\{(a, b) \in S \times S \mid(\forall e \in E(S))\right.$ ea $\left.\mathcal{L}^{*} e b\right\}$ is the largest congruence on $S$ contained in $\mathcal{L}^{*}$. The dual result holds for $\eta_{r}$;

(3) $\eta=\eta_{l} \cap \eta_{r}$ is the largest congruence on $S$ contained in $\mathcal{H}^{*}$.

For a type-B semigroup $S, \eta$ is the largest idempotent-separating good congruence on $S$ contained in $\mathcal{H}^{*}$. We can define the least cancellative congruence $\sigma$ on a type-B semigroup $S$, as follows:

$(\forall a, b \in S) \quad a \sigma b \Longleftrightarrow(\exists e \in E(S)) \quad(e a e=e b e)$ (see, [11]).

Next, we recall some of the basic facts about the fuzzy relations. Let $X$ be a non-empty set. A fuzzy relation on $X$ is a map $\mu: X \times X \longrightarrow[0,1]$.

Definition 1.4. [13] Let $\mu$ and $\nu$ be fuzzy relations on a semigroup $S$. Then the product $\mu \circ \nu$ of $\mu$ and $\nu$ is defined by $\mu \circ \nu(a, b)=\vee_{x \in S}[\mu(a, x) \wedge \nu(x, b)]$ for all $a, b \in S$, and $\mu \subseteq \nu$ is defined by $\mu(x, y) \leq \nu(x, y)$ for all $x, y \in S$.

As in [13], a fuzzy relation $\mu$ on a semigroup $S$ is called fuzzy equivalent if $\mu(a, a)=1, \mu(a, b)=\mu(b, a)$ and $\mu \circ \mu \subseteq \mu$ for all $a, b \in S$. A fuzzy relation $\mu$ on a semigroup $S$ is called compatible if $\mu(a x, b x) \geq \mu(a, b)$ and $\mu(x a, x b) \geq \mu(a, b)$ for all $a, b, x \in S$. Let $\mu$ be a fuzzy equivalence relation on a semigroup $S$. For each $a \in S$, we define a fuzzy subset $\mu_{a}$ of $S$ as follows: $\mu_{a}(x)=\mu(a, x)$ for all $x \in S$. A fuzzy equivalence relation $\mu$ on $S$ which is compatible is called a fuzzy congruence on $S$. A fuzzy congruence $\mu$ on $S$ is idempotent-separating [resp. idempotent-pure] if for all $e, f \in E(S), a \in S$ the equality $\mu_{e}=\mu_{f}\left[\operatorname{resp} . \mu_{a}=\mu_{e}\right]$ implies $e=f[\operatorname{resp} . a \in E(S)]$. We denote by $C_{\rho}$ the characteristic function of a binary relation $\rho$ on $S$. Then, as is easily seen, $\rho$ is a congruence on $S$ if and only if $C_{\rho}$ is a fuzzy congruence on $S$. Let $\mu$ be a fuzzy congruence on a semigroup $S$. Then $S / \mu=\left\{\mu_{a} \mid a \in S\right\}$. Define the binary operation " *" on $S / \mu$ as follows: $\mu_{a} * \mu_{b}=\mu_{a b}$. We can prove that $S / \mu$ is a semigroup with respect to the binary operation $*$.

Lemma 1.5. [13] Let $\mu$ be a fuzzy equivalence relation on a semigroup $S$. Then

(1) for all $a, b \in S, \mu_{a}=\mu_{b} \Longleftrightarrow \mu(a, b)=1$;

(2) if $\mu$ is a fuzzy congruence on $S$, then $\mu^{-1}=\{(a, b) \in S \times S: \mu(a, b)=1\}$ is a congruence on $S$.

Definition 1.6. [12] A fuzzy congruence $\mu$ on an abundant semigroup $S$ is called a fuzzy good congruence on $S$ if $\mu_{a} \mathcal{L}^{*}(S / \mu) \mu_{a^{*}}$ and $\mu_{a} \mathcal{R}^{*}(S / \mu) \mu_{a^{+}}$ for all $a \in S$.

Evidently, if $\mu$ is a fuzzy good congruence on an abundant semigroup $S$, then $S / \mu$ is an abundant semigroup with respect to the binary operation $*$. In 
particular, if $S / \mu$ is a cancellative semigroup with respect to the binary operation $*$, then we call $\mu$ a fuzzy cancellative congruence. For brevity, we denote by $\left(\mu_{a}\right)^{+}\left[\operatorname{resp} .\left(\mu_{a}\right)^{*}\right]$ a typical idempotent $\mathcal{R}^{*}$-related [ resp. $\mathcal{L}^{*}$-related ] to $\mu_{a}$.

The following lemma is due to Li and Liu in [12].

Lemma 1.7. [12] Let $S$ be an abundant semigroup. Then

(1) $\rho$ is a good congruence on $S$ if and only if $C_{\rho}$ is a fuzzy good congruence on $S$;

(2) $\mu^{-1}$ is a (resp. an idempotent-separating) good congruence on $S$ if and only if $\mu$ is a (resp. an idempotent-separating) fuzzy good congruence on $S$;

(3) if $\mu$ is a fuzzy good congruence on $S$ such that $\mu \subseteq C_{\mathcal{H}^{*}}$, then $\mu$ is an idempotent-separating fuzzy good congruence on $S$;

(4) if $E(S)$ is a left quasi-normal band (i.e., $(\forall x, y, z \in E(S)) x y z=x y x z)$, then $(\forall a \in S) \mu_{a} \in E(S / \mu) \Longleftrightarrow(\exists e \in E(S)) \mu_{a}=\mu_{e}$.

Corollary 1.8. Let $\mu$ be a fuzzy good congruence on an adequate semigroup S. Then

(1) $(\forall a \in S) \mu_{a} \in E(S / \mu) \Longleftrightarrow(\exists e \in E(S)) \mu_{a}=\mu_{e}$;

(2) $S / \mu$ is an adequate semigroup with respect to the binary operation *;

(3) for any $a \in S, \mu_{a^{+}}=\left(\mu_{a}\right)^{+}$and $\mu_{a^{*}}=\left(\mu_{a}\right)^{*}$;

(4) $(\forall e \in E(S), a \in S) \mu_{e a} \in E(S / \mu) \Longrightarrow \mu_{a e a} \in E(S / \mu)$;

(5) $(\forall e \in E(S), a \in S) \mu_{a e} \in E(S / \mu) \Longrightarrow \mu_{a e a} \in E(S / \mu)$.

Proof. (1) It follows from Lemma $1.7(4)$ since $E(S)$ is a semilattice ( i.e., the elements of $E(S)$ are commutative ).

(2)Obviously, $S / \mu$ is an abundant semigroup with respect to the binary operation $*$. To verify that $S / \mu$ is adequate, let $\mu_{a}, \mu_{b} \in E(S / \mu)$. Then, by $(1)$, there are idempotents $e, f \in S$ such that $\mu_{a}=\mu_{e}$ and $\mu_{b}=\mu_{f}$. Note that $E(S)$ is a semilattice. We have $e f=f e$. Thus $\mu_{a} * \mu_{b}=\mu_{e} * \mu_{f}=\mu_{e f}=\mu_{f e}=$ $\mu_{f} * \mu_{e}=\mu_{b} * \mu_{a}$, as required.

(3)It follows from (2).

(4) Let $\mu_{e a} \in E(S / \mu)$, where $e \in E(S), a \in S$. Then, by (1), there exists $f \in E(S)$ such that $\mu_{e a}=\mu_{f}$. Hence, $\mu_{f}=\mu_{e a}=\mu_{e e a}=\mu_{e} * \mu_{e a}=\mu_{e} * \mu_{f}=$ $\mu_{e f}=\mu_{f e}$, and so $\mu_{e a a}=\mu_{e a} * \mu_{a}=\mu_{f} * \mu_{a}=\mu_{f e} * \mu_{a}=\mu_{f} * \mu_{e} * \mu_{a}=\mu_{f} * \mu_{e a}=$ $\mu_{f} * \mu_{f}=\mu_{f}$. Therefore, $\mu_{\text {aea }} * \mu_{\text {aea }}=\mu_{a} * \mu_{e a} * \mu_{a} * \mu_{e a}=\mu_{a} * \mu_{\text {eaa }} * \mu_{e a}=$ 
$\mu_{a} * \mu_{f} * \mu_{e a}=\mu_{a} * \mu_{e a} * \mu_{f}=\mu_{a} * \mu_{e a}=\mu_{a e a}$ since $E(S / \mu)$ is a semilattice, as required.

(5)It is a similar proof of (4).

Proposition 1.9. Let $\mu$ be a fuzzy good congruence on an adequate semigroup $S$. Then $\mu$ is fuzzy cancellative if and only if $\mu_{e}=\mu_{f}$ for all $e, f \in E(S)$.

Proof: We only need to prove the converse part, because the direct part can be easily seen. For this, let $\mu_{a}, \mu_{b}, \mu_{c} \in S / \mu$ be such that $\mu_{a} * \mu_{b}=\mu_{a} * \mu_{c}$. Since $\mu$ is a fuzzy good congruence on $S$, we have $\mu_{a^{*}} * \mu_{b}=\mu_{a^{*}} * \mu_{c}$. On the other hand, by the hypothesis, we have $\mu_{a^{*}}=\mu_{b^{+}}=\mu_{c^{+}}$. Thus $\mu_{b^{+}} * \mu_{b}=\mu_{c^{+}} * \mu_{c}$, that is, $\mu_{b}=\mu_{c}$. Therefore, $\mu$ is fuzzy left cancellative. Similarly, we can get that $\mu$ is fuzzy right cancellative. This completes the proof.

\section{$2 \quad$ Fuzzy good congruences on type-B semi- groups}

In this section, we shall consider fuzzy good congruences on type-B semigroups.

Lemma 2.1. Let $\mu$ be a fuzzy good congruence on a type-B semigroup $S$ and $a, b \in S$. Then the following statements are true:

(1) $a \leq b \Longrightarrow \mu_{a} \leq \mu_{b}$;

(2) $\mu_{a} \leq \mu_{b} \Longrightarrow \mu_{a^{+}} \leq \mu_{b^{+}}, \mu_{a^{*}} \leq \mu_{b^{*}}$.

Proof. (1)It follows immediately from the definition of " $\leq$ " in Lawson [9].

(2)By Corollary 1.8(1), $E(S / \mu)=\left\{\mu_{e} \mid e \in E(S)\right\}$. Let $\mu_{a}, \mu_{b} \in S / \mu$ such that $\mu_{a} \leq \mu_{b}$. Then $\mu_{a}=\mu_{e} * \mu_{b}=\mu_{b} * \mu_{f}$, where $e, f \in E(S)$. That is, $\mu_{a}=\mu_{e b}=\mu_{b f}$. Hence, by Corollary 1.8(3), $\mu_{a^{+}}=\left(\mu_{a}\right)^{+}=\left(\mu_{e b}\right)^{+}=\mu_{(e b)^{+}}=$ $\mu_{e b^{+}}=\left(\mu_{b f}\right)^{+}=\mu_{(b f)^{+}}=\mu_{b^{+}(b f)^{+}}$since $S$ is $\mathcal{R}^{*}$-unipotent (i.e., each $\mathcal{R}^{*}$-class contains exactly one idempotent). Thus, $\mu_{a^{+}}=\mu_{e} * \mu_{b^{+}}=\mu_{b^{+}} * \mu_{(b f)^{+}}$. That is, $\mu_{a^{+}} \leq \mu_{b^{+}}$. Similarly, we can prove that $\mu_{a^{*}} \leq \mu_{b^{*}}$.

Theorem 2.2. Let $\mu$ be a fuzzy good congruence on a type-B semigroup $S$. Then $S / \mu$ is a type-B semigroup with respect to the binary operation *.

Proof. Let $\mu$ be a fuzzy good congruence on $S$. Then, by Corollary 1.8(2), $S / \mu$ is an adequate semigroup with respect to the binary operation $*$. Next, we prove that $S / \mu$ is type-B. To see this, let $\mu_{e}, \mu_{f} \in E(S / \mu)$ and $\mu_{a} \in S / \mu$, 
where $e, f \in E(S)$. Then

$$
\begin{array}{rlrl}
\left(\mu_{e} * \mu_{f} * \mu_{a}\right)^{*} & =\left(\mu_{e f a}\right)^{*}=\mu_{(e f a)^{*}} & & \text { ( by Corollary 1.8(3)) } \\
& =\mu_{(e a)^{*}(f a)^{*}} & & \text { (since } S \text { satisfies Condition (B1)) } \\
& =\mu_{(e a)^{*} * \mu_{(f a)^{*}}} & & \\
& =\left(\mu_{e a}\right)^{*} *\left(\mu_{f a}\right)^{*} & & (\text { by Corollary 1.8(3)) } \\
& =\left(\mu_{e} * \mu_{a}\right)^{*} *\left(\mu_{f} * \mu_{a}\right)^{*} . &
\end{array}
$$

This gives that $S / \mu$ satisfies Condition (B1). On the other hand, let $\mu_{a} \in$ $S / \mu, \mu_{e} \in E(S / \mu)$ such that $\mu_{e} \leq\left(\mu_{a}\right)^{*}$, where $e \in E(S)$. Then, by Corollary 1.8 (3), $\mu_{e} \leq \mu_{a^{*}}$. That is, $\mu_{e}=\mu_{e} * \mu_{a^{*}}=\mu_{e a^{*}}$. But $e a^{*} \leq a^{*}$. We get that $e a^{*}=(f a)^{*}$ for some $f \in E(S)$ since $S$ satisfies Condition (B2). Hence, $\mu_{e}=\mu_{e a^{*}}=\mu_{(f a)^{*}}=\left(\mu_{f a}\right)^{*}=\left(\mu_{f} * \mu_{a}\right)^{*}$, where $\mu_{f} \in E(S / \mu)$, and thus $S / \mu$ satisfies Condition (B2). Therefore, $S / \mu$ is right type-B. Dually, we can prove that $S / \mu$ is left type-B. This completes the proof.

Theorem 2.3. Let $S$ be a type-B semigroup. Then the following statements are true:

(1) $C_{\eta}$ is an idempotent-separating fuzzy good congruence on $S$ such that $C_{\eta} \subseteq C_{\mathcal{H}^{*}}$

(2) $C_{\sigma}$ is the least fuzzy cancellative congruence on $S$;

(3) If $\mu$ is a fuzzy good congruence on $S$, then $\mu$ is a fuzzy cancellative congruence on $S$ if and only if $C_{\sigma} \subseteq \mu$.

Proof. (1) It follows from Lemma 1.7 (1) and Lemma 1.7 (3).

(2)Obviously, $\sigma$ is a good congruence on $S$. By Lemma 1.7(1), we see that $C_{\sigma}$ is a fuzzy good congruence on $S$. Next, we prove that $C_{\sigma}$ is a fuzzy cancellative congruence on $S$. For this, let $\left(C_{\sigma}\right)_{a},\left(C_{\sigma}\right)_{b},\left(C_{\sigma}\right)_{c} \in S / C_{\sigma}$ be such that $\left(C_{\sigma}\right)_{a} *\left(C_{\sigma}\right)_{b}=\left(C_{\sigma}\right)_{a} *\left(C_{\sigma}\right)_{c}$, that is, $\left(C_{\sigma}\right)_{a b}=\left(C_{\sigma}\right)_{a c}$. Then, by Lemma $1.5(1)$, we have $C_{\sigma}(a b, a c)=1$, that is, $(a b, a c) \in \sigma$. But $\sigma$ is a cancellative congruence on $S$, so $(b, c) \in \sigma$. Thus $C_{\sigma}(b, c)=1$. By Lemma 1.5(1), we get that $\left(C_{\sigma}\right)_{b}=\left(C_{\sigma}\right)_{c}$. This means that $C_{\sigma}$ is a fuzzy left cancellative congruence on $S$. Similarly, we can get that $C_{\sigma}$ is a fuzzy right cancellative congruence on $S$.

Finally, suppose that $\mu$ is any fuzzy cancellative congruence on $S$. Let $a$ and $b$ be any elements of $S$. If $(a, b) \in \sigma$, then $C_{\sigma}(a, b)=1$. By the definition of $\sigma$, we have $e a e=e b e$ for some $e \in E(S)$. Thus $\mu_{e a e}=\mu_{e b e}$, that is, $\mu_{e} * \mu_{a} * \mu_{e}=$ $\mu_{e} * \mu_{b} * \mu_{e}$. As $\mu$ is fuzzy cancellative, it follows that $\mu_{a}=\mu_{b}$. Hence, by Lemma $1.5(1)$, we get that $\mu(a, b)=1$. If $(a, b) \notin \sigma$, then $C_{\sigma}(a, b)=0 \leq \mu(a, b)$. Consequently, $C_{\sigma} \subseteq \mu$, that is, $C_{\sigma}$ is the least fuzzy cancellative congruence on $S$. 
(3) Clearly, we only need to prove the converse part. Let $e, f \in E(S)$. Then, by (2) and Proposition 1.9, we get that $\left(C_{\sigma}\right)_{e}=\left(C_{\sigma}\right)_{f}$. Thus, by Lemma 1.5(1), we have $C_{\sigma}(e, f)=1$. But $C_{\sigma} \subseteq \mu$, so $\mu(e, f)=1$. By Lemma 1.5(1), we have $\mu_{e}=\mu_{f}$. Therefore, by Proposition $1.9, \mu$ is a fuzzy cancellative congruence on $S$.

Theorem 2.4. Let $\mu$ be a fuzzy good congruence on a type-B semigroup $S$ and let $\mu^{-1}=\{(a, b) \in S \times S \mid \mu(a, b)=1\}$. Then $\mu$ is a fuzzy cancellative congruence on $S$ if and only if $\sigma \subseteq \mu^{-1}$.

Proof. Necessity. Let $a, b \in S$ be such that $(a, b) \in \sigma$. Then there exists an idempotent $e$ of $S$ such that eae $=e b e$. Thus $\mu_{e a e}=\mu_{e b e}$, that is, $\mu_{e} * \mu_{a} * \mu_{e}=$ $\mu_{e} * \mu_{b} * \mu_{e}$. Since $\mu$ is a fuzzy cancellative congruence on $S$, we have $\mu_{a}=\mu_{b}$. By Lemma 1.5(1), we get that $\mu(a, b)=1$. Hence $(a, b) \in \mu^{-1}$. Therefore, $\sigma \subseteq \mu^{-1}$.

Sufficiency. Let $a$ and $b$ be any elements of $S$. If $(a, b) \in \sigma$, then $C_{\sigma}(a, b)=$ 1. On the other hand, by the assumption, we have $(a, b) \in \mu^{-1}$. Thus, $\mu(a, b)=$ 1. If $(a, b) \notin \sigma$, then $C_{\sigma}(a, b)=0 \leq \mu(a, b)$. Therefore, $C_{\sigma} \subseteq \mu$. Hence, it follows from Theorem 2.3(3), that $\mu$ is a fuzzy cancellative congruence on $S$.

\section{References}

[1] S. Armstrong, The structure of type-A semigroups. Semigroup Forum. 29(1984), 319-336.

[2] K. A. Dib, N. Galhum, Fuzzy ideals and fuzzy bi-ideals in fuzzy semigroups. Fuzzy Sets and Systems. 92(1997), 103-111.

[3] A. El-Qallali, J. B. Fountain , Idempotent-connected abundant semigroups. Proc. Roy. Soc. Edinburgh Sect. A 91(1981), 79-90.

[4] J. B. Fountain, Adequate semigroups. Proc. Edinburgh Math. Soc. 22(1979), 113-125.

[5] J. B. Fountain, Abundant semigroups, Proc. London Math Soc. 44 (1982) 103-129.

[6] X. J. Guo, K. P. Shum, On translational hulls of type-A semigroups. Joural of Algebra. 269(2003), 240-249.

[7] J. M. Howie. An introduction to semigroup theory. Academic Press. London. 1976.

[8] N. Kuroki. Fuzzy congruences on inverse semigroups. Fuzzy Sets and Systems. 87(1997), 335-340. 
[9] M. V. Lawson, The natural partial order on an abundant semigroup. Proc. Edinburgh Math. Soc. 30(1987), 169-186.

[10] C. H. Li, X.J. Guo. Good congruences on perfect rectangular bands of adequate semigroups, advances in mathematics, 38(2009), 465-476.

[11] C. H. Li, L. M. Wang, On the translational hull of a type-B semigroup. Semigroup Forum, 82(2011),516-529.

[12] C. H. Li, E. G. Liu, Fuzzy good congruences on left semi-perfect abundant semigroups. Communications in Algebra, 39(2011),3090-3103.

[13] J. N. Mordeson, D. S. Malik and N. Kuroki. Fuzzy semigroups. Springerverlag Berlin Heidelberg New York, 2003.

[14] V. Murali. Fuzzy equivalence relations. Fuzzy Sets and Systems. 30(1989), 155-163.

[15] W. C. Nemitz. Fuzzy relations and fuzzy functions. Fuzzy Sets and Systems. 19(1986), 177-191.

[16] M. Petrich, Completely regular semigroups. New York, Jhon Wiley and Sons Inc. 1999.

[17] M. Petrich, Inverse Semigroups. Wiley, NewYork, 1984.

[18] A. Rosenfeld, Fuzzy groups, J. Math. Anal. Appl. 35 (1971) 512-517.

[19] M. Samhan, Fuzzy Congruences on semigroups. Inform. Sci. 74(1993),165175.

[20] Y. J. Tan. Fuzzy congruences on regular semigroups, Fuzzy Sets and Systems 117 (2001) 447-453.

[21] X. Y. Xie, On prime quasi-prime, weakly quasi-prime fuzzy left ideals of semigroup, Fuzzy Sets and Systems 123 (2001) 239-249.

[22] X. Y. Xie, Fuzzy Rees congruences on semigroups, Fuzzy Sets and Systems 102 (1999) 353-359.

\section{Received: December 7, 2013}

